\title{
Study of magnetoresistance of epitaxial magnetite films grown on vicinal MgO (100) substrate
}

\author{
R. G. S. Sofin, S. K. Arora, and I. V. Shvets \\ SFI Nanoscience Laboratories, Department of Physics, Trinity College Dublin, Dublin 2, Ireland
}

(Presented on 10 November 2004; published online 6 May 2005)

\begin{abstract}
The magnetoresistance (MR) studies of magnetite thin films deposited on vicinal $\mathrm{MgO}$ substrates show an enhanced MR along the miscut direction as compared to the direction perpendicular to it. The MR anisotropy increases with the decrease in temperature and peaks at the Verwey transition. The increase in magnetoresistance and anisotropy behavior is attributed to the formation of a greater number of out-of-plane shifted antiphase boundaries due to the step edges on vicinal $\mathrm{MgO}$ substrates. These local electronic and spin structure modifications at the APBs introduce additional spin scattering and are responsible for the increase in MR along the miscut. (C) 2005 American Institute of Physics. [DOI: 10.1063/1.1850361]
\end{abstract}

\section{INTRODUCTION}

Recently, there has been considerable interest in the growth of magnetic thin films on vicinal surfaces owing to the intriguing physics involved and technological potential. ${ }^{1-4}$ The studies on vicinal surfaces have been carried out mainly to understand the effect of lateral modulation of the electronic structure induced by step edges on the magnetic anisotropies. ${ }^{3,4}$ In our previous publication we have shown that the growth of Fe on stepped Mo (110) substrates leads to the formation of self-assembled nanowedges of size 20-40 $\mathrm{nm}$ and a switching of the magnetic easy axis from along the step edges to direction perpendicular to them. ${ }^{3}$ There are few reports on the study of magnetoresistance properties of thin films grown on vicinal surfaces. ${ }^{5-7}$ However, there are no reports on the study of magnetoresistance or resistivity anisotropy of half metallic ferromagnetic (HMFM) oxides.

Magnetite $\left(\mathrm{Fe}_{3} \mathrm{O}_{4}\right)$ is one of the important HMFM oxide due to its high Curie temperature $(858 \mathrm{~K})$ and associated Verwey transition at $\sim 120 \mathrm{~K} .{ }^{8}$ Epitaxial $\mathrm{Fe}_{3} \mathrm{O}_{4}$ films grown on $\mathrm{MgO}$ are known to suffer from the formation of antiphase boundaries (APBs). ${ }^{9-12}$ APBs are formed due to the differences in translational (in-plane shifted APBs) and rotational (out-of-plane shifted APBs) symmetries between $\mathrm{Fe}_{3} \mathrm{O}_{4}$ and MgO. ${ }^{11,12}$ These APBs act as sharp antiferromagnetic boundaries for the spin polarized transport and contribute to magnetoresistance observed in epitaxial $\mathrm{Fe}_{3} \mathrm{O}_{4}$ films. ${ }^{13,14}$ In this article we present the magnetoresistance results obtained for epitaxial $\mathrm{Fe}_{3} \mathrm{O}_{4}$ films grown on vicinal $\mathrm{MgO}$. The $\mathrm{MgO}$ substrate has a $2^{\circ}$ miscut along $\langle 011\rangle$ direction off the (100) plane. The vicinal nature of the substrate acts as a template to grow epitaxial $\mathrm{Fe}_{3} \mathrm{O}_{4}$ films with out-of-plane shifted APBs predominantly aligned along the step edges rather than perpendicular to them. The miscut direction (MC) is perpendicular to the step edges. The orthogonal in-plane direction is perpendicular to the miscut (PMC) and is along the terrace step edges. Additional spin scattering produced by the local structural and spin disorders at the APBs enhances the MR along the MC direction. A comparison of the MR behavior of $\mathrm{Fe}_{3} \mathrm{O}_{4}$ film on vicinal (large miscut angle) and low vicinal (small miscut angle) is also provided.

\section{EXPERIMENT}

The $\mathrm{Fe}_{3} \mathrm{O}_{4}$ thin films were grown on (100) oriented $\mathrm{MgO}$ single crystal substrates using an oxygen plasma assisted molecular beam epitaxy (MBE) system (DCA MBE M600, Finland) with a base pressure $5 \times 10^{-10}$ Torr. Details of the film growth procedure are given elsewhere. ${ }^{15}$ In situ reflection high energy electron diffraction (RHEED) was used to confirm epitaxial growth and also the growth mode. From the RHEED intensity oscillations it was found that the films grew in a layer-by-layer mode (at a rate of $0.3 \AA / s$. In the present study we report results obtained on two representative samples named, sample A and sample B, in which the $\mathrm{MgO}(100)$ substrates have nominal miscut of $0.5^{\circ}\left( \pm 0.05^{\circ}\right)$ and $2^{\circ}\left( \pm 0.05^{\circ}\right)$ along $\langle 011\rangle$ directions. Structural characterization of the samples was performed using a high-resolution $\mathrm{X}$-ray diffractometer (HRXRD, Bede-D1, UK). Miscut of the samples was determined from the variation in Bragg angle for the symmetric diffraction for different azimuth.

Electrical resistivity and magnetoresistance (MR) measurements were carried out in the standard dc four probe collinear configuration. A variable field (up to $2 \mathrm{~T}$ ) permanent magnet (Model: MM 2000 26.5-001, Multimag Magnetic Solutions Ltd., Ireland) was used to apply magnetic fields in the desired orientation. The MR is defined as;

$$
M R \%=\left[\frac{R(H)-R(0)}{R(0)}\right] \times 100,
$$

where $R(H)$ and $R(0)$ are the resistance of the sample with and without field, respectively. To determine the magnetoresistance anisotropy, the measurements were carried out by passing current in two directions, which are orthogonal to each other, i.e., along and perpendicular to the direction of MC. 


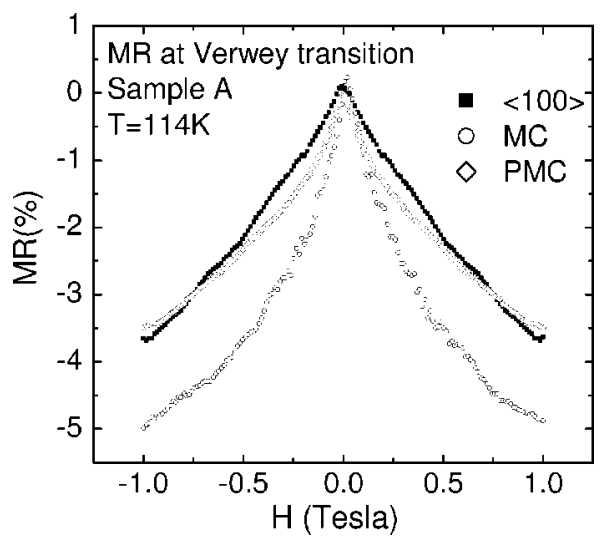

FIG. 1. Magnetoresistance of the low miscut sample (sample A) measured at Verwey transition $(T=114 \mathrm{~K})$ in the $\langle 010\rangle$, MC and PMC directions for the in-plane magnetic field parallel to the direction of current.

\section{RESULTS AND DISCUSSION}

The structural characterization of the $\mathrm{Fe}_{3} \mathrm{O}_{4}$ thin films grown on $\mathrm{MgO}$ substrates was performed using HRXRD. From the symmetric $(400 / 200)$ and asymmetric $(622 / 311)$ Bragg reflections the in-plane lattice constant of $\mathrm{Fe}_{3} \mathrm{O}_{4}$ thin film was found to be twice $(0.8426 \mathrm{~nm})$ the lattice constant of the $\mathrm{MgO}$ substrate. The out-of-plane lattice constant was found to be $0.8372 \mathrm{~nm}$. The full width at half maximum for the thin film peaks of the symmetric (400/200) reflections were 0.044 and 0.077 degrees for sample A and sample B, respectively. The broadening of the thin film peak for sample $\mathrm{B}$ is due to its smaller thickness. In situ RHEED and ex situ HRXRD characterization show that the films grew pseudomorphically and maintained a one to one registry with the $\mathrm{MgO}$ substrate. The unit cell volume, which was consistent with the bulk magnetite, indicates that the films were stoichiometric.

Sample A showed a Verwey transition at $114 \mathrm{~K}$. This was grown on a low vicinal substrate. The miscut angle of this sample was $0.45^{\circ}$ directed essentially along the $\langle 011\rangle$ direction (just $3^{\circ}$ off from the $\langle 011\rangle$ ), which was measured using HRXRD. Figure 1 shows the MR plots of sample A at $114 \mathrm{~K}$ (the Verwey transition region) with current passing along $\langle 010\rangle, \mathrm{MC}$, and PMC directions. In all cases the magnetic field was applied in the film plane and parallel to the current direction. The MR at $1 \mathrm{~T}$ field along the $\mathrm{MC}$ direction was found to be $4.98 \%$, which is $1.3 \%$ and $1.5 \%$ higher than the MR along the $\langle 010\rangle$ and PMC directions, respectively. The difference in MR between MC and PMC directions is not due to crystalline anisotropic effect, since the two orthogonal directions (MC and PMC directions) in a cubic crystal are identical and electronic transport properties along them should remain identical. This confirms that the observed enhancement in MR is purely due to the effect of the presence of step edges which form a template for increasing the density of antiferromagnetically coupled antiphase boundaries running preferentially along the PMC.

The APBs in the $\mathrm{Fe}_{3} \mathrm{O}_{4} / \mathrm{MgO}$ heteropitaxy are related to shift vectors that could be in-plane or out-of-plane shift vectors. ${ }^{11,12}$ APBs with out-of-plane shift vectors on a $\mathrm{MgO}$ substrate can be produced across the regions separated by an

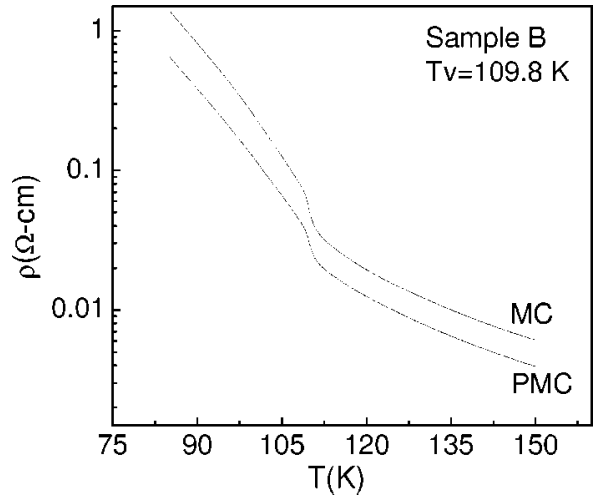

FIG. 2. Resistivity as a function of temperature measured along and perpendicular to the miscut direction for the high miscut sample sample B [45 nm thick $\mathrm{Fe}_{3} \mathrm{O}_{4}$ film on $\mathrm{MgO}(100)$ substrate with $2^{\circ}$ miscut along the $\langle 011\rangle$ direction].

atomic step with a height of half the lattice constant of $\mathrm{MgO}$. By growing $\mathrm{Fe}_{3} \mathrm{O}_{4}$ on vicinal $\mathrm{MgO}$ (100) substrate [e.g., tilted a few degrees from high symmetry planes (001), with a vicinal angle along $\langle 011\rangle$ direction], one can expect a greater number of APBs formed with out-of-plane shifts across the steps $(\langle 011\rangle$ direction). These out-of-plane shifted APBs are formed when two nucleation sites from adjacent terraces coalesces. In addition to the enhancement in MR it is also expected to have a higher resistivity in the direction of $\mathrm{MC}$ than perpendicular to it. It is clear that the greater the miscut angle, the greater the density of anisotropic APBs.

Figure 2 shows resistivity variation with temperature measured along and perpendicular to the miscut direction for a $45 \mathrm{~nm}$ thick $\mathrm{Fe}_{3} \mathrm{O}_{4}$ film grown on $\mathrm{MgO}$ (100) oriented substrate (sample $\mathrm{B}$ ), which has a miscut of $2^{\circ}$ along the $\langle 011\rangle$ direction. The resistivity across the step edges is about 2 times larger than the resistivity along the step edges. This suggests that the presence of a higher density of APBs along the $\mathrm{MC}$ direction enhances the charge carrier scattering and reduces the conductivity along the $\mathrm{MC}$ direction as compared to the PMC direction. The Verwey transition temperatures for both the directions were found to be $109.8 \mathrm{~K}$. The magnetization measurements gave a saturation magnetization of $480 \mathrm{emu} / \mathrm{cm}^{3}$ and $454 \mathrm{emu} / \mathrm{cm}^{3}$ in sample A and sample $\mathrm{B}$, respectively. The reduced magnetization in sample $\mathrm{B}$ also confirms that it has a higher density of APBs than sample A. Figures 3(a) and 3(b) shows the MR of sample B for three different temperatures viz, $300 \mathrm{~K}, 130$ and $109.8 \mathrm{~K}$. In the vicinity of the Verwey transition, the MR values show a clear difference between the MC and PMC directions. With a decrease in temperature the MR is found to increase and shows peaks at the Verwey transition for both the directions. However, the magnitude of the MR along the MC remains substantially higher than that along the PMC direction. The larger MR observed along the MC direction is attributed to the step edges induced additional spin scattering of charge carriers. The maximum value of MR, observed in the Verwey transition region, was $12.34 \%$ at $2 \mathrm{~T}$ field and $10.05 \%$ at $1 \mathrm{~T}$ field, which is $5.42 \%$ and $5.19 \%$ higher than the corresponding values observed in the PMC direction. The MR at $1 \mathrm{~T}$ field for sample B is found to be $4.03 \%$ higher than that of the low miscut sample. MR at other temperatures also shows 


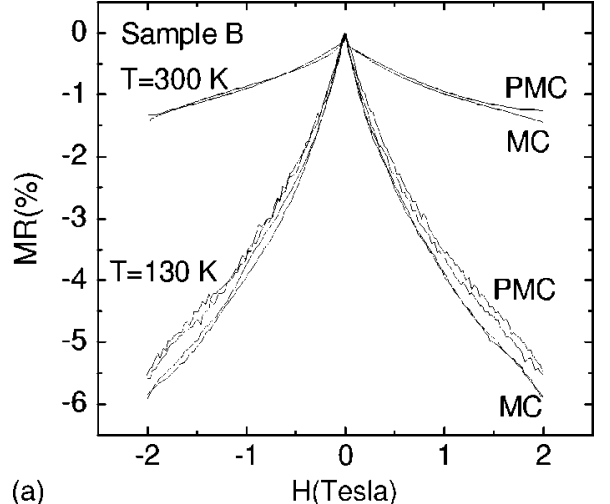

(a)

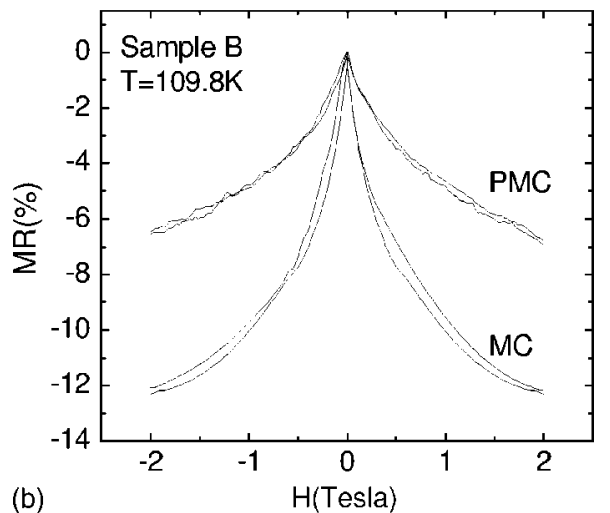

(b)

FIG. 3. Magnetoresistance of the sample B measured at (a) 300 and $130 \mathrm{~K}$ and (b) $109.8 \mathrm{~K}$ for the MC and PMC directions for the in-plane magnetic field parallel to the direction of current.

the same behavior. The observed value of MR is higher than any of the previously reported MR values for epitaxial magnetite films. ${ }^{13,14,16}$ Another noticeable feature is that there is a significant enhancement in the low field MR. The MR shows a steep rise up to a field of $0.7 \mathrm{~T}$ and for higher magnetic fields $(>0.7 \mathrm{~T}))$ there is a crossover to shallower field dependence. This points out the fact that at higher fields the moments coupled antiferromagnetically (AFM) across the APBs are tending towards a saturation value. These observations are in line with the predictions of Evetts et al. ${ }^{17}$ model of spin disorder induced scattering at the grain boundaries in epitaxial bicrystal junctions.

The observed changes in MR with temperature can be qualitatively understood from the fact that the APBs introduce additional spin scattering due to the local structural and spin disorder. This modifies the transfer integral $t_{\mathrm{ij}}$ that governs the electronic transport between $\mathrm{Fe}^{2+}$ and $\mathrm{Fe}^{3+}$ octahedral cations. Furthermore, the strain field in the vicinity of APBs is altered which influences the spin transport across them. These periodic perturbations in the electronic structure along the MC direction produce greater contribution of spin dependent scattering than that along the PMC direction. The peak in MR observed in the vicinity of the Verwey transition results from abrupt changes in the thermodynamic quantities accompanied with the first order Verwey transition. Similar peak structure was previously reported in bulk and thin films of magnetite. ${ }^{18,19}$

\section{CONCLUSION}

Magnetoresistance properties of epitaxial $\mathrm{Fe}_{3} \mathrm{O}_{4}$ films on vicinal $\mathrm{MgO}$ substrate show a close correlation with the direction of the step edges. The MR is significantly higher in the direction of MC due to enhanced spin scattering induced by the out-of plane shifted APBs formed at the step edges. The low field MR along the MC direction is enhanced significantly in comparison with the PMC direction or films grown on nonvicinal substrates. The crossover from a strong to shallower field dependence of MR in high fields is explained in terms of alignment of AFM moments at the APBs in the field direction.

\section{ACKNOWLEDGMENTS}

The financial support from the Science Foundation Ireland is gratefully acknowledged (Project No. 00/PI.1/C042).

${ }^{1}$ B. B. Maranville, A. L. Shapiro, F. Hellman, D. M. Schaadt, and E. T. Yu, Appl. Phys. Lett. 81, 517 (2002).

${ }^{2}$ B. Degroote, M. Major, J. Meersschaut, J. Dekoster, and G. Langouche, Surf. Sci. 482-485, 1090 (2001).

${ }^{3}$ V. Usov, S. Murphy, and I. V. Shvets, J. Appl. Phys. 95, 7312 (2004).

${ }^{4}$ F. Naguyen-Van-Dau, M. Sissaiu, A. Schuhl, and P. Galtier, J. Appl. Phys. 81, 4482 (1997).

${ }^{5}$ M. A. M. Gijs, M. T. Johnson, A. Reinders, P. J. Huisman, R. J. M. van de Veerdonk, S. K. J. Lenczowski, and R. M. J. van Gansewinkel, Appl. Phys. Lett. 66, 1839 (1995).

${ }^{6}$ M. Sussiau, F. Nguyen Van Dau, P. Galtier, A. Encinas, and A. Schuhl, J. Magn. Magn. Mater. 165, 1 (1997).

${ }^{7}$ S. Sugahara and M. Tanaka, Appl. Phys. Lett. 80, 1969 (2002).

${ }^{8}$ F. Walz, J. Phys.: Condens. Matter 14, 285 (2002).

${ }^{9}$ D. T. Margulies, F. T. Parker, F. E. Spada, R. S. Goldman, J. Li, R. Sinclair, and A. E. Berkowitz, Phys. Rev. B 53, 9175 (1996).

${ }^{10}$ F. C. Voogt, T. T. M. Palstra, L. Niesen, O. C. Rogojanu, M. A. Janes, and T. Hibma, Phys. Rev. B 57, 08107 (1998).

${ }^{11}$ D. T. Margulies, F. T. Parker, M. L. Rudee, F. E. Spada, J. N. Chapman, R. A. Aitchison, and A. E. Berkowitz, Phys. Rev. Lett. 79, 5162 (1997).

${ }^{12}$ W. Eerenstein, T. T. M. Palstra, and T. Hibma, Phys. Rev. B 68, 014428 (2003).

${ }^{13}$ W. Eerenstein, T. T. M. Palstra, S. S. Saxena, and T. Hibma, Phys. Rev. Lett. 88, 247204 (2002).

${ }^{14}$ M. Ziese, R. Hohne, N. H. Hong, J. Dienelt, K. Zimmer, and P. Esquinazi, J. Magn. Magn. Mater. 242-245, 450 (2002).

${ }^{15}$ Y. Zhou, X. Jin, and I. V. Shvets, J. Appl. Phys. 95, 7357 (2004).

${ }^{16}$ M. Ziese, R. Hohne, H. C. Semmelhack, K. H. Han, P. Esquinazi, and K. Zimmer, J. Magn. Magn. Mater. 279, 331 (2004).

${ }^{17}$ J. E. Evetts, M. G. Blamire, N. D. Mathur, S. P. Isaac, B. S. Teo, L. F. Cohen, and L. Macmanus-Driscoll, Philos. Trans. R. Soc. London, Ser. A 356, 1593 (1998).

${ }^{18}$ S. B. Ogale, K. Ghosh, R. P. Sharma, R. L. Greene, R. Ramesh, and T. Venkatesan, Phys. Rev. B 57, 7823 (1998).

${ }^{19}$ V. V. Gridin, G. R. Hearne, and J. M. Honig, Phys. Rev. B 53, 15518 (1996). 